\title{
Can mRNA Vaccines Turn the Tables During the COVID-19 Pandemic? Current Status and Challenges
}

\author{
Deva Priya Sagili Anthony ${ }^{1} \cdot$ Kavitha Sivakumar $^{1} \cdot$ Priyanka Venugopal $^{1} \cdot$ Damal Kandadai Sriram $^{2} \cdot$ Melvin George $^{1}$
}

Accepted: 6 March 2021 / Published online: 23 March 2021

(c) The Author(s), under exclusive licence to Springer Nature Switzerland AG 2021, corrected publication 2021

\begin{abstract}
The COVID-19 pandemic continues to affect millions of people across the world. The current global statistics for the disease are 111 million cases and 2.45 million deaths, with new cases emerging each day. Although several drugs including remdesivir have been approved for emergency use, they remain ineffective in bringing the infection under control. Therefore, there is a need for highly effective and safe vaccines against COVID-19. The recent advancements in mRNA vaccines have catapulted them to be forefront in the race to develop vaccines for COVID-19. Two mRNA vaccines, BNT162b2 and mRNA-1273, developed by Pfizer-BioNTech and Moderna Therapeutics, respectively, have been granted authorization for emergency use by the US Food and Drug Administration. Interim analysis of the clinical trials for BNT162b2 and mRNA1273 vaccines reported an efficacy of $95 \%$ and $94.1 \%$, respectively, after the second dose. The adverse events for both the vaccines have been found to be mild to moderate, with mostly injection-site reactions and fatigue. No serious adverse events have been reported. Moreover, Pfizer-BioNTech and Moderna Therapeutics have announced that their vaccines are effective even against the new strains (B.1.17 and B.1.351) of the virus. Both companies are now scaling up the production of the vaccines to meet the global demand. Although the long-term efficacy, safety, and immunogenicity of these vaccines is uncertain, there is hope that they can turn the tables against COVID-19 in this current pandemic situation.
\end{abstract}

\section{Key Points}

The BNT162b2 vaccine by Pfizer-BioNtech has been found to have an efficacy of $95 \%$.

The mRNA-1273 vaccine by Moderna therapeutics has shown an efficacy of $94.1 \%$.

Neither vaccine has exhibited any serious adverse events and both have been found to be safe.

The BNT162b2 and mRNA-1273 vaccines are effective against the new strains of COVID-19, B.1.17 and B.1.351.

Deva Priya Sagili Anthony, Kavitha Sivakumar, Priyanka Venugopal: Equal contribution (joint first authors).

Melvin George

drmelvingeorge@hindumissionhospital.org

1 Department of Clinical Research, Hindu Mission Hospital, Tambaram, Chennai 600 045, India

2 Diabetology and Endocrinology, Hindu Mission Hospital, Tambaram, Chennai 600 045, India

\section{Introduction}

The COVID-19 pandemic, which has affected over 111 million people and resulted in the death of approximately 2.45 million people globally to date, is far from over [1]. Countries in Europe and the USA are embroiled in controlling the second wave of new COVID-19 infections [2, 3]. Several drugs have been evaluated as possible therapeutic solutions for COVID-19 but remain ineffective [4]. Even remdesivir, one of the few novel medications previously approved by the US Food and Drug Administration (FDA) for emergency use authorization (EUA), could not demonstrate robust efficacy in the SOLIDARITY trial, leading to the drug being considered by the World Health Organization (WHO) as having limited value [4]. With no cure in sight, the only solution available at the moment is to continue with preventive measures such as hand washing, social distancing, avoiding crowds, and wearing masks [5]. Hence, the seemingly only possible solution to the current situation is to develop an effective vaccine against COVID-19 [6]. Messenger RNA (mRNA) is a single-stranded RNA molecule that is involved in the transcription process or gene expression. mRNA is made from DNA via transcription that 
occurs in the nucleus of the cell. It is then transported to the cytoplasm for protein synthesis, a process called translation. The role of mRNA is to carry messages from the nucleus to the cytoplasm, hence, the name "messenger" RNA [7]. In other words, mRNA carries genetic information encoded in DNA to be used by cells for synthesizing specific proteins essential for various cellular processes [8]. Preclinical studies since the 1990s have reported major advancements in mRNA therapeutics, especially in terms of mRNA manufacturing and delivery mechanisms [9]. In the current pandemic scenario, mRNA vaccines seem to be the solution due to their rapid and flexible production as compared to other vaccine types. In addition, the prior disadvantage of using mRNA vaccines due to their inefficient cellular uptake by the host cells and intracellular degradation by RNAases has been overcome by encapsulating the mRNA into nanoparticles [10]. Scientists and co-founders of the biotechnology firm, BioNTech, Dr. Uger Sahin and Dr. Ozlem Tureci, were the first to lay the foundations for the development of an mRNA vaccine against COVID-19. During the onset of the pandemic, they partnered with the US firm Pfizer to rapidly test the BNT162b2 vaccine in clinical trials [11]. While it would normally take 3-4 years to develop conventional viral vaccines, Pfizer-BioNTech developed the vaccine against COVID-19 within 10 months (after the genetic sequence of the virus was published), using RNA technology [12]. According to sources, Moderna Therapeutics claimed to have developed its mRNA-1273 vaccine against COVID19 within 2 days after the genetic sequence was revealed by China. The vaccine was apparently manufactured and shipped to the National Institutes of Health for initiating the Phase I clinical trial just a month after the first death was reported in the USA, thus showing the fastest timeline for vaccine development in history [13]. This article is a brief review of the current status of the two mRNA vaccines that are in an advanced phase of development for COVID19 - BNT162b2 by Pfizer-BioNtech and mRNA-1273 by Moderna Therapeutics.

\section{Mechanism of Action of mRNA Vaccines in Fighting COVID-19}

The main function of mRNA vaccines is to deliver the gene of interest, encoding the specific target antigen into the host cytoplasm for the production of proteins (including membrane, secretory, or intracellular) [14]. mRNA vaccines consist of two types, namely non-replicating mRNA or "NRM" (the most common and conventional type) and self-amplifying mRNA (SAM). The difference between the two types is that the former encodes only the protein antigen, whereas the latter, apart from encoding the protein, also facilitates
RNA replication. Although NRM is predominant, SAM vaccines have recently been employed in different phases of clinical trials for COVID-19 [15]. One of the major issues with mRNA vaccines has been their stability, due to their susceptibility to degradation by RNAases. Several approaches have been employed to overcome the instability and potential immunogenicity of the vaccines, including molecular stabilization. This strategy involves engineering the sequences and structure of the mRNA to improve its stability and thereby extend its half-life. The modifications include elongation of the poly (A) tail, $5^{\prime}$ cap modification, structure of the untranslated regions (UTRs) and sequence patterns in the open reading frame (ORF), and incorporation of modified nucleotides [15]. The in vitro transcribed (IVT) mRNA is produced from linear DNA transcripts using T7, T3, or Sp6 phage RNA polymerase and comprises UTRs (at $5^{\prime}$ and $3^{\prime}$ ends) flanking the coding sequence, i.e., gene/protein of interest, a 5' cap, and a 3' poly-A tail [16]. The structure of IVT mRNA for the candidate vaccine is illustrated in Fig. 1. The resultant IVT mRNA should ideally resemble a complete mature mRNA molecule. Upon administration of mRNA vaccines, the IVT mRNA will be released from its carriers and into the cytoplasm of the host cell, where ribosomes facilitate translation of the mRNA into the surface spike proteins, which are then presented to antigen-presenting cells (APCs), which in turn elicit an immune response. The primary features of candidate mRNA vaccines are listed in Table 1. Some of the advantages of employing mRNAs for vaccination are that they are non-infectious, can be rapidly manufactured, are cost-effective, and are safe. However, due to their nature of easily disintegrating and a lack of efficient internalization by host cells, mRNA vaccines demand highly reliable and efficient delivery systems. Encapsulation of the vaccine by delivery systems or carriers comprises various types like peptides, polymers, nanoparticles (especially lipids), naked mRNA in solution and ex vivo via APCs like dendritic cells [17].

\section{3 mRNA Vaccines for COVID-19}

As mentioned, BNT162b2 and mRNA-1273 are manufactured by Pfizer-BioNTech and Moderna Therapeutics, respectively. Both these vaccines are administered intramuscularly and involve a similar mechanism of action (Fig. 2). Both the vaccines are encapsulated by lipid nanoparticles (LNPs) as their delivery system, which contributes to easier permeability and uptake by the host cells [18, 19]. However, Moderna Therapeutics has modified the LNP formulation of their mRNA-1273 vaccine to improve the stability of the vaccine. This is the primary reason why their mRNA vaccine is more durable and stable at usual refrigeration (stored at $-20{ }^{\circ} \mathrm{C}$ for 6 months, $2-8{ }^{\circ} \mathrm{C}$ for 1 month, or normal room 
Fig. 1 Structure of the in vitro mRNA vaccine. $m R N A$ messenger RNA, LNP lipid nanoparticle, UTR untranslated region, $C D S$ coding sequence, $I V T$ in vitro transcribed, $G$ guanine, $A$ adenine

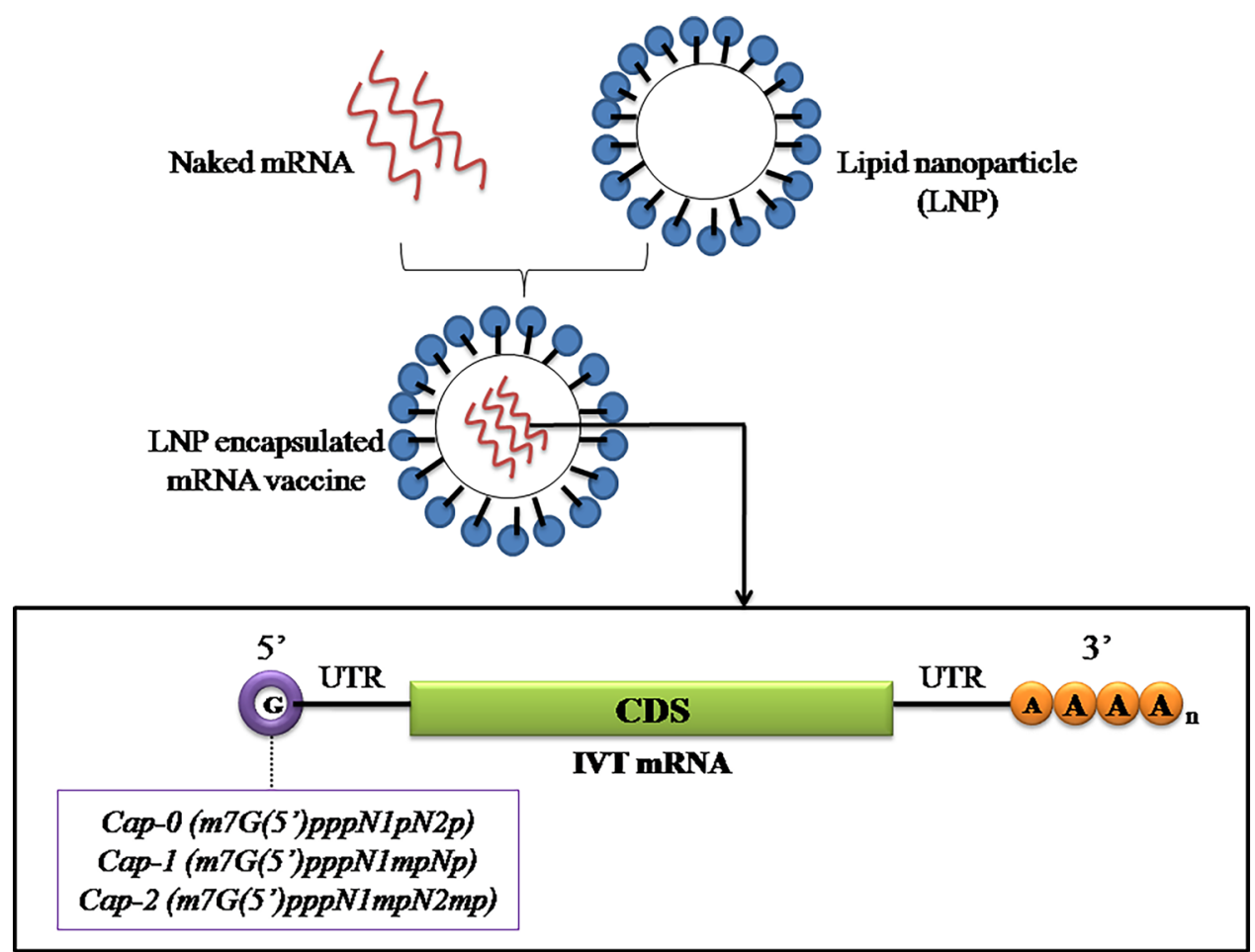

Table 1 Features of IVT mRNA and their functions that influence the potential of an ideal vaccine

\begin{tabular}{|c|c|}
\hline Component & Function \\
\hline $5^{\prime}$ cap & Prevents mRNA degradation from exonucleases and innate immune sensing \\
\hline $3^{\prime}$ poly-A tail & $\begin{array}{l}\text { Influences translation and protein expression. Protects mRNA from degrada- } \\
\text { tion by preventing decapping }\end{array}$ \\
\hline 5' UTR & $\beta$-Globin enhances translation efficiency \\
\hline 3' UTR & $\alpha$-Globin improves stability of mRNA; $\beta$-globin enhances translation efficiency \\
\hline CDS/coding sequence & Modification of sequence contribute to improved expression \\
\hline
\end{tabular}

UTR untranslated region, $C D S$ coding sequence, $I V T$ in vitro transcribed, $m R N A$ messenger RNA

temperature for $12 \mathrm{~h}$ ) when compared to Pfizer's BNT162b2 vaccine (stored at $-75{ }^{\circ} \mathrm{C}$ for 6 months, $2-8{ }^{\circ} \mathrm{C}$ for 5 days, or room temperature for up to $2 \mathrm{~h}$ ) [19].

\section{Efficacy of mRNA Vaccine Candidates}

\subsection{BNT162b2 Vaccine}

The Phase III clinical trial conducted between 27 July 2020 and 14 November 2020 recruited around 44,820 participants, and after screening, 43,548 participants were randomized at 152 sites globally including the USA, Germany, South Africa, Brazil, Turkey, and Argentina. Among these, 43,448 participants were injected with the BNT162b2 vaccine $(21,720)$ or placebo $(21,728)$. All the participants were $\geq 16$ years of age, with a median age of 52 years, and around $42 \%$ of the study population was $>55$ years. The participants belonged to different ethnicities, with the majority of them being White (83\%) and the rest Hispanic (28\%) and African Americans (9\%). A total of eight participants were confirmed COVID-19 cases (symptomatic) with onset of 7 days post second dose of the vaccine injection, thereby suggesting an efficacy of $95 \%$ for the BNT162b2 vaccine. Meanwhile, among the placebo group 162 participants were found to be confirmed COVID-19 cases [20]. The observed efficacy of $95 \%$ is significantly higher than the minimal efficacy standard of at least $50 \%$, which was set by the FDA in their guidelines for COVID-19 vaccine development [21]. The trial data reported efficacy for symptomatic cases, whereas there was no information about the ability of the vaccine to prevent asymptomatic COVID-19 infection. This end-point is being evaluated and once the trial is completed, these results are expected. This information is especially 
Fig. 2 Schematic representation of the mechanism of action of the mRNA vaccine. $S A R S$ $\mathrm{CoV}-2$ severe acute respiratory syndrome coronavirus $2, m R N A$ messenger RNA, $S$ proteins spike proteins, $A P C$ antigenpresenting cell

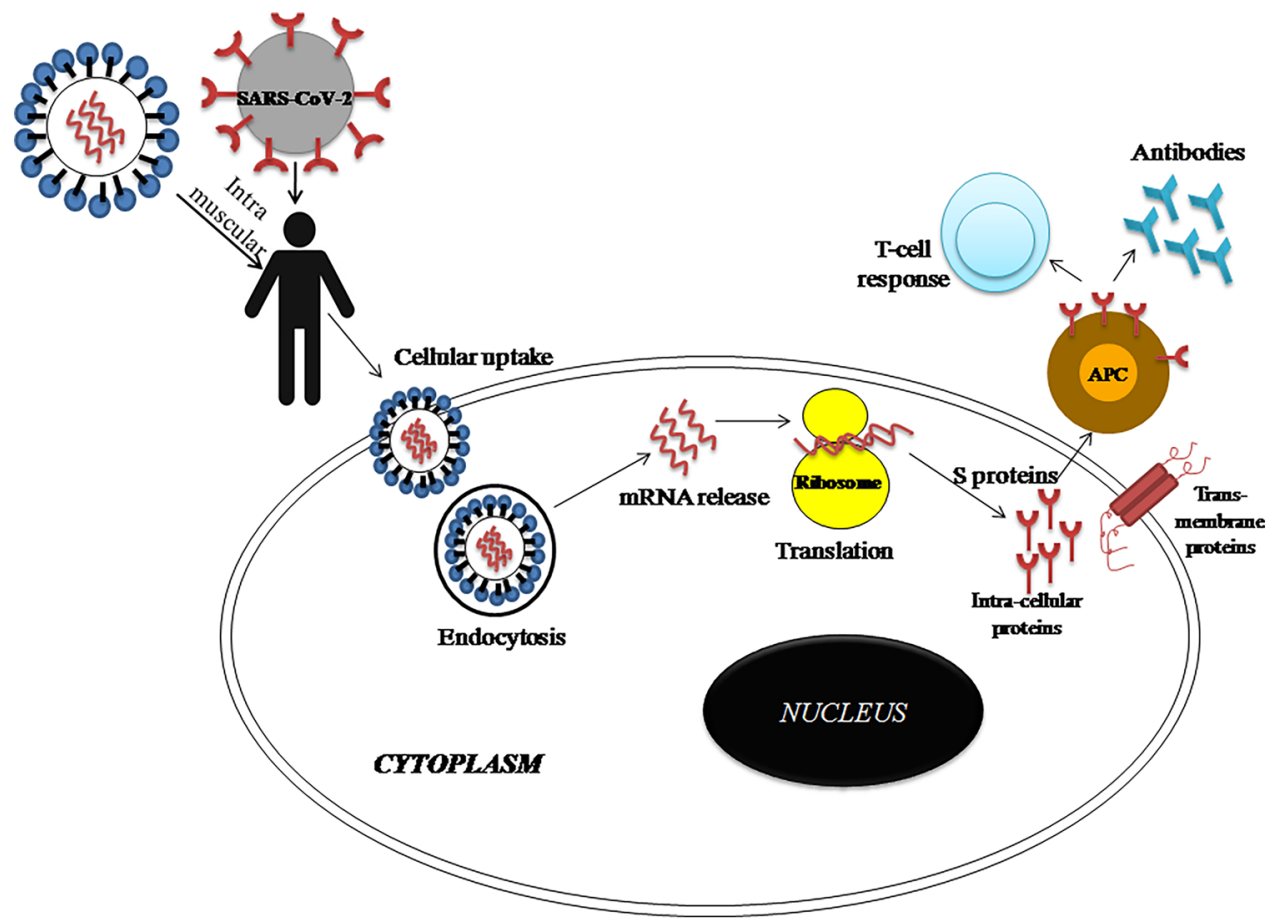

pertinent as the eradication of the virus can effectively only be achieved if the numbers of asymptomatic carriers are decreased as much as the symptomatic cases are [22].

\section{2 mRNA-1273 Vaccine}

The preclinical studies carried out with rhesus macaque models showed that mRNA-1273 is able to induce a greater antibody response than that seen with convalescent-phase serum from patients with COVID 19 at both $10 \mu \mathrm{g}$ as well as $100 \mu \mathrm{g}$ dosages, with titer values of 501 and 3481, respectively. These vaccines were also able to induce a CD4 T cell response that was type-1 helper T-cell based as well as Th2 or CD8 T-cell responses. Animals in the vaccine group did not exhibit viral replication in the bronchoalveolar fluid following challenge with plaque-forming units of SARS COV-2 injected in the intra-tracheal route [23]. A Phase I doseescalation study was performed in 45 healthy volunteers in whom mRNA-1273 was administered as three doses of 25, 100 , and $250 \mu \mathrm{g}$ at 0 and 28 days. Volunteers in the $250 \mu \mathrm{g}$ group had the highest antibody geometric mean titer compared with those in the $100 \mu \mathrm{g}$ and $25 \mu \mathrm{g}$ groups $(213,526$, 109,209 , and 40,227, respectively) after the first dose. All three dosage groups had an increase in antibody titers following the second dose of the vaccine and the titer values for the $25 \mu \mathrm{g}, 100 \mu \mathrm{g}$, and $250 \mu \mathrm{g}$ groups were 299,751 , 782,719 , and 1,192,154, respectively [24].

The Coronavirus Efficacy or COVE Phase III trial was initiated in July 2020 to determine the efficacy and safety of the mRNA-1273 vaccine against COVID-19. This is a randomized, placebo-controlled trial in which 30,420 participants were enrolled and administered either the vaccine or placebo in a 1:1 ratio, with 15,210 participants in each group. All the participants were given their first dose of injection (placebo/100 $\mu$ g vaccine) between the period of 27 July 2020 and 23 October 2020 . The trial was performed at 99 sites in the USA and according to the International Council for Harmonization of Technical Requirements for Pharmaceuticals for Human Use, Good Clinical Practice Guidelines and all applicable government regulations. More than $96 \%$ of participants received the second dose. The primary endpoint of the trial was defined as prevention of COVID-19 with onset at least 14 days after the second injection in participants without any prior SARS-CoV-2 infection. The vaccine exhibited $94.1 \%$ efficacy, with 185 and 11 participants in the placebo and mRNA-1273 groups, respectively, developing symptomatic COVID-19 illness. The baseline demographics did not differ among the two groups and the mean age of the participants was 51.4 years. There was no significant gender bias, with $47.3 \%$ of participants being females. Age distribution of the participants revealed $24.8 \%$ belonged to the $\geq 65$-year group and $16.7 \%$ to the $<65$-year group, with predisposing medical conditions and at high risk for severe COVID-19. Racial and ethnicity backgrounds of the participants were found to be predominantly White (79.2\%), with the rest being African American and Hispanic. This trial did not have sufficient data for evaluating asymptomatic infection; however, assessment of asymptomatic infection and viral shedding are still in process [24]. 


\section{Safety of mRNA Vaccine Candidates}

\subsection{BNT162b2 Vaccine}

In the early Phase I/Phase II trials [25], the most common local reactions were pain at the injection site. In the group receiving the BNT162b1 vaccine at a dosage of $10 \mu \mathrm{g}, 58.3 \%$ experienced local injection-site reactions while all the participants in the $30 \mu \mathrm{g}$ and $100 \mu \mathrm{g}$ groups had injection-site reactions. In the placebo arm, only $22 \%$ had injection-site reactions. None of these reactions were of severe intensity. Even after the second dose, the most common reaction continued to be only local injection-site reactions with an almost similar frequency. Fatigue, headache, and fever were the other adverse events observed in the study population. Biochemical and hematological investigations did not reveal any significant abnormalities besides a transient reduction in the lymphocyte count. The lymphocytopenia resolved and returned to normal after 6-8 days. On the second dose, grade 2 neutropenia was observed in one participant each in the $10 \mu \mathrm{g}$ and $30 \mu \mathrm{g}$ groups after $6-8$ days of vaccination. These two participants were followed up and no adverse events of neutropenia have been reported to date $[26,27]$. The participants who received the BNT162b2 vaccine reported more local reactions than those receiving placebo.

In the Phase III safety analysis, the most commonly reported local reaction was pain at the injection site within 7 days after injection, with less than $1 \%$ of participants across all age groups reporting severe pain. Participants older than 55 years reported pain less frequently (71\% reported pain after the first dose; $66 \%$ after the second dose) than did younger participants (83\% after the first dose; $78 \%$ after the second dose). Fatigue and headache were the most commonly reported systemic events (59\% and 52\%, respectively, after the second dose among younger vaccine recipients; $51 \%$ and $39 \%$ among older recipients), although fatigue and headache were also reported by many placebo recipients ( $23 \%$ and $24 \%$, respectively, after the second dose among younger vaccine recipients; $17 \%$ and $14 \%$ among older recipients). The severity of all the events ranged from mild to moderate and was found to be resolved within 1 or 2 days. Two deaths were reported in the vaccine group and four deaths were observed in the placebo group. None of the deaths were related to the vaccination. Overall, the safety profile of the BNT162b2 vaccine was found to be reassuring [27].

\section{2 mRNA-1273 Vaccine}

The safety of the mRNA-1273 vaccine was reviewed weekly by a protocol safety review and the safety monitoring board systematically. The first interim analysis of the trial determined that the vaccine fulfilled the pre-specified efficacy criteria. The assessment included both solicited (local and systemic events for 7 days after each injection) and unsolicited adverse events (28 days after each injection). The solicited local adverse events consisted of injection-site reactions of grade 1 or 2 severity, which were observed predominantly in the mRNA1273 vaccine group rather than the placebo group. They lasted for an average of 2.6 days after the first dose and 3.2 days after the second dose. The delayed local adverse events were characterized by erythema, induration, and tenderness, which lasted for 4-5 days. Systemic adverse events were also found to be more common among the vaccine group when compared to the placebo group, affecting about $54.9 \%$ and $79.4 \%$ of participants after first and second dose, respectively. The systemic events were also observed in the placebo group, affecting $42.2 \%$ of participants after the first dose and $36.5 \%$ of participants after the second dose. These events consisted of fever, headache, fatigue, myalgia, arthralgia, chills, nausea, and vomiting, which persisted for an average of 2.9 days after the first dose and 3.1 days after the second dose. All the adverse events were observed to be only mild to moderate in severity. Unsolicited and serious adverse events were reported 28 days after the dose, which included three fatalities in the placebo group and two fatalities in the vaccine group, none of which were related to the vaccine. The only adverse events related to the trial were fatigue and headache, which were observed in both groups. All the adverse events were generally short-lived. Altogether, the safety of mRNA-1273 appears to be convincing [24].

\section{Challenges of mRNA Vaccines}

Most of the knowledge about mRNA vaccines exists only with regard to cancer. mRNA vaccines for infectious diseases are still a work in progress. Recent studies have shown mRNA to be efficient for vaccination. Although they are reported as highly safe, efficient, rapid, and cost-effective, naked mRNAs are easily degraded by extracellular RNAases and lack efficient uptake by the host cells [15]. Hence, they require several in vitro and in vivo transfection reagents to improve cellular uptake and also protect the mRNA from degradation. Researchers have now developed a new technology in the mRNA vaccine platform for COVID-19, where they have employed LNP as carriers of mRNA vaccines, which deliver it to the host cytoplasm. The main challenge with mRNA vaccines is stability. Encapsulation of these mRNA vaccines by LNPs facilitate their stability and permeability. Another important challenge is to maintain the balance between immune and inflammatory responses, to accomplish an optimal risk-versus-benefit profile of the vaccine [14]. Upon synthesis of the spike proteins, they bind to the APCs and elicit T-cell responses and antibodies. Any unnecessary immune or inflammatory response is harmful 
to the host. Therefore, careful administration and dosing of the mRNA vaccines are mandatory to stimulate the optimal response. Asymptomatic COVID-19-infected individuals are silent carriers of the infection and are a major cause of the pandemic. An estimated range of $18-81 \%$ of infections are asymptomatic, which contributes greatly to the spread of COVID-19 [28]. The trial data of the two vaccines did not provide information on their efficacy in preventing asymptomatic infection. Since asymptomatic infection seems to be a vital factor in the spread of the disease, these vaccines should be able to prevent not only the symptomatic infection, but also asymptomatic COVID-19. However, to date there have been no reports on this end-point. With the increasing number of cases worldwide, scaling up the vaccine production is essential. Scaling up depends on the capacity, supply chain, and technical challenges. Meeting the global demand for the vaccines is the most challenging aspect, and requires good infrastructure for speeding up the manufacturing and the potential to meet the temperature-sensitive necessities [29]. Pfizer-BioNtech has now announced that they will be boosting the vaccine production to two billion doses this year by expanding their manufacturing units and increasing their suppliers and contract manufacturers [30]. Moderna has teamed up with Lonza Group AG to ramp up the production of their vaccine [31]. The BNT162b2 vaccine requires storage at ultra-low temperatures, for which deepfreezers are essential to avoid compromising the integrity of the vaccine. To overcome these thermostability issues, Pfizer-BioNTech has come up with three options for storage. These include a commercially available ultra-low-temperature freezer that can extend the shelf-life for up to 6 months, specially designed temperature-controlled thermal shippers, and refrigeration units commonly available in hospitals. The thermal shippers can maintain the $-70{ }^{\circ} \mathrm{C} \pm 10{ }^{\circ} \mathrm{C}$ environment for 10 days (with the package unopened) and for 30 days (with an opened package) by re-icing once at 5 days [32]. Moderna, on the other hand, has an edge over PfizerBioNTech since its vaccine can be stored in a conventional fridge (at $2-8{ }^{\circ} \mathrm{C}$ ) for up to 30 days and at $-20{ }^{\circ} \mathrm{C}$ for up to 6 months [33]. Another major challenge is to manage the cost of these vaccines, since it will be difficult for some underdeveloped and developing nations to purchase them. In order to overcome this, certain organizations such as the Global Alliance for Vaccines and Immunization (GAVI) are bringing together public and private sectors to ensure equal accessibility to all nations world-wide irrespective of their income levels. COVAX is a global initiative organized by GAVI, Coalition for Epidemic Preparedness Innovations (CEPI), and the WHO to ensure equal access of the COVID-19 vaccines to low- and lower-middle-income countries. Pfizer-BioNTech has recently signed an agreement with COVAX to supply up to 40 million doses in 2021 at a not-for-profit price, which will be supplied to 92 low- and lower-middle-income countries. The first delivery of the vaccine is expected to be in the first quarter of 2021. In addition, Pfizer-BioNTech are planning to partner with various other global health organizations to strengthen the healthcare system by assessing the supply chain in low-income countries in order to overcome the transportation and storage issues of the vaccine. They are also coordinating with international agencies for distributing the vaccines to refugee and vulnerable populations [34]. Meanwhile, COVAX is still in discussion with Moderna Therapeutics for equitable access to the vaccine [35].

The new strains of COVID-19, namely B.1.1.7, B.1.351, and P.1, were first identified in the UK, South Africa, and Brazil, respectively. All three strains were found to be more infectious when compared to the parent strain, particularly the B.1.1.7 variant, which was reported to be the most common and lethal type. These strains spread more rapidly and their mutations allow them to escape the immune system. This in turn challenges the treatment and management of the disease [36]. WHO has reported that B.1.1.7 and B.1.351 did not alter the effectiveness of mRNA-1273 vaccine [37]. Similarly, the BNT162b2 vaccine has also been reported to be highly effective against the new variants. However, recent findings revealed that both the vaccines had reduced activity to neutralize the B.1.351 variant. Pfizer-BioNTech and Moderna Therapeutics are planning on updating their vaccines and developing booster doses to improve their efficacy against any future variants $[38,39]$. Several new variants are being reported sporadically and will continue to emerge due to the nature of RNA viruses, particularly coronavirus. When several mutations arise leading to newer strains, he effectiveness of the vaccines may be affected. Hence, the genetic sequence of the new variants needs to be constantly investigated as it evolves [40].

\section{Misconceptions of mRNA Vaccines}

There is a misconception that nucleic acid vaccines can alter the genetic makeup of the host. This might be true for DNA vaccines, but not for mRNA vaccines. DNA vaccines are transported into the nucleus of the cell, where they are transcribed into mRNAs, which then enter the cytoplasm and stimulate the production of antigens [41]. Since DNA vaccines enter the nucleus of the cell, there is a risk of permanently altering the genetic makeup of the host cell [42]. Meanwhile, mRNA vaccines do not enter the nucleus. Instead, they enter the cytoplasm of the host and undergo translation and post-translational modifications to produce antigens. So, their role is only to instruct the cell to make spike proteins and trigger antibody production, thereby promoting an immune response. Therefore, the mRNA vaccines 
do not pose any threat with regard to altering the host's genetic material [43, 44].

\section{Other mRNA Vaccines in the Pipeline}

This review highlights the two mRNA vaccines BNT162b2 and mRNA-1273, which are approved for emergency use by the FDA. Apart from these two vaccines, there are various other mRNA vaccines in different phases of clinical trials, as shown in Table 2 [45]. Of these vaccines, HGC019 and ARCT-021 (LUNAR-COV19) are categorized as selfamplifying mRNA vaccines, whereby they carry alpha viral replicase for genetic replication, in addition to the gene of interest. This enables the production of high levels of the $S$ protein [46]. However, a major disadvantage of these types of vaccines is the production of unwanted aggressive inflammation reactions, which can be harmful to the host. Gennova Biopharmaceuticals in Pune, India and HDT Bio (a Seattlebased company) has employed a novel and advanced delivery system called LION, which possesses the adjuvant property of improving the immune response of the vaccine. In addition, it has been shown to enhance the storage stability of the vaccine as well as improve permeability and bioavailability, and decrease the adverse effects of the vaccine [47].

\section{The Regulatory Status of mRNA Vaccines}

Pfizer and BioNtech have combined manufacturing networks, and had the capacity to provide 50 million vaccine doses globally in 2020 and up to 1.3 billion doses by the end of 2021 (subject to clinical success, manufacturing capacity, and regulatory approval or authorization) [48]. They have to face more obstacles in distributing the vaccine all over the world. The vaccine needs to be refrigerated at under $-70{ }^{\circ} \mathrm{C}$, resulting in the company building boxes that will maintain the temperature of the vaccine. The BNT162b2 vaccine was approved in the UK for emergency use on 2 December 2020 [49]. It was also approved in Bahrain on 5 December 2020; this was the second country to approve the Pfizer-BioNTech vaccine, followed by the UK [50]. Canada approved the Pfizer-BioNtech vaccine on 9 December 2020; 249,000 doses were to be available by the end of December and 4 million doses by March 2021 [51]. In a trial conducted in the USA, four cases of Bell's palsy were reported among 21,720 participants who received the Pfizer-BioNTech vaccine. US regulators have, however, dismissed the fear about Bell's palsy, as the incidence is consistent with how common the viral reaction is in random populations. Twenty to 30 cases of Bell's palsy are observed among 100,000 people in the UK each year [52]. The FDA approved this vaccine for emergency use in preventing COVID-19 on 11 December 2020. The company expects to file a Biologics License Application for regulatory approval by next year [53].

Based on the Phase III interim efficacy and safety data of the mRNA-1273 vaccine against COVID-19, the FDA granted Emergency Use Authorization on 11 December 2020 [53, 54]. Moderna Therapeutics, on 1 December 2020, sent an application to the European Medicines Agency (EMA) for Conditional Marketing Authorization (CMA) of its mRNA vaccine candidate [30], following which, the company partnered with Roche to utilize the Elecsys Anti-SARS COV-2 antibody test in its upcoming trial of the mRNA-1273 vaccine candidate. This test is an immunoassay for the qualitative in vitro selection of antibodies (including IgG) to SARS COV-2. The FDA granted EUA for the test on 25 November 2020 [55].

Moderna has ensured that the storage, handling, and distribution of this vaccine can be carried out with the available infrastructure at a global level. The storage of this vaccine makes it more suitable for distribution in the rural areas as they can remain stable for 6 months at a temperature of $-20^{\circ} \mathrm{C}$ and for 30 days in a medical refrigerator [56].

Japan approved the BNT162b2 vaccine on 14 February 2021. A shipment of 400,000 doses from Pfizer's factory in Belgium arrived in Japan on 12 February 2021 [57]. They are expected to receive 144 million doses from Pfizer, 120 million

Table 2 mRNA vaccines currently undergoing clinical trials

\begin{tabular}{llll}
\hline S. no & Name of vaccine & Manufacturer & Status \\
\hline 1 & MRT5500 & Sanofi and Translate Bio & Preclinical \\
2 & HGC019 & Gennova Biopharmaceuticals and HDT Bio & Phase I/II \\
3 & ARCoV & Academy of Military Medical Sciences, Suzhou Abogen & Phase I \\
& & Biosciences, Walvax Biotechnology & Phase I \\
4 & ChulaCoV19 & Chula Vaccine Research Centre & Phase I \\
5 & PTX-COVID19-B & Providence Therapeutics & Phase II \\
6 & ARCT-021 (LUNAR-COV19) & Duke-NUS/Arcturus Therapeutics & Phase III \\
7 & CVnCoV & CureVac & \\
\hline
\end{tabular}

mRNA messenger RNA 
doses from Astra Zeneca, and 50 million doses from Moderna by the end of this year. The first shots will be given to around 20,000 front-line workers at hospitals starting by the middle of this month, followed by other medical staff and geriatric people in April 2021 [58]. In Israel, about 520,000 people have been vaccinated, which covers $27 \%$ of their population. The Maccabi Healthcare services, one of the Health Maintenance Organizations in Israel, reported the vaccine to be $93 \%$ effective at preventing COVID-19. Even though 15 people were hospitalized and four presented with serious illnesses, there were no fatalities. A $0 \%$ virus death was reported in people after immunization [59].

On 10 February 2021 the mRNA-1273 vaccine was approved for EUA in Qatar, following approval of the BNT162b2 vaccine [60]. In Singapore, the mRNA-1273 vaccine was approved in February 2021, after approval for BNT162b2 in December 2020. The first shipment of mRNA1273 vaccines is expected around March 2021, whereas BNT162b2 vaccine's first shipment was received on 21 December 2020. As of 2 February 2021, over 175,000 people have received their first dose of BNT162b2 and around 6000 people have received their second dose. Furthermore, BNT162b2 has been approved in other parts of Southeast Asia, such as the Philippines and Malaysia. The National Pharmaceutical Regulatory Agency of Malaysia has granted conditional approval for the vaccine and the first batch is expected to arrive on 26 February 2021 [61]. On 12 February 2021, the FDA granted approval to Moderna for increasing their vaccine formula by $40 \%$ into each vial shipment, which will now be able to pack 14 doses into each vial instead of 10 doses [62].

\section{COVID-19 New Variants: An Emerging Concern}

During the second wave of the pandemic, multiple variants of the SARS-CoV-2 virus were detected globally. The variant forms arise due to mutations in the virus, which normally occur over time, and these variants may either disappear or persist. Scientists are currently working on the genetics of the virus to help understand the consequences of such variants in terms of the spread of the infection and symptoms and severity of the infected individuals. The strain B.1.1.7 was detected in the UK during the fall of 2020 and in the USA in late December, 2020. The variant possesses a large number of mutations and was reported to spread rapidly among various populations. Another strain, named B.1.351, was first identified in South Africa in October 2020 and later in December 2020 in the USA. Although this variant occurred independently, it shares a few mutations with the B.1.1.7 variant. The P.1 variant was first detected in some Brazilian travelers at a Japanese airport in early January 2021, and possesses additional mutations, making the strain unrecognizable by antibodies. This variant was identified in the USA in late January 2021 [63]. According to the New and Emerging Respiratory Virus Threats Advisory Group (NERVTAG) based in the UK, B.1.1.7, also known as the Kent variant, may be approximately $70 \%$ more deadly when compared to other strains [64]. Recently, the Centers for Disease Control has reported around 1523, 21, and five cases with the B.1.17, B.1.351, and P.1 variants, respectively, across the US (Ref: https://www.cdc.gov/coronavirus/2019ncov/transmission/variant-cases.html). The South African variant (B.1.351) was first identified in India in November 2020 [65]. Around 192 COVID-19 cases belonging to the new strains were identified in early February 2021, which included four cases from the South African variant and one from the Brazilian strain (P.1) [66]. Evidence from preliminary reports suggests that the new strains, especially the B.1.1.7 variant, exhibit firm binding to cells due to their sticky nature, which is caused by mutations in the spike protein [40]. The symptoms of COVID-19 from the new strains have included fever, cough, sore throat, myalgia, and fatigue, all which were similar to the original strain but are more persistent [64]. Scientists are still investigating the new strains to understand the spread, transmission, and severity of the disease when compared to the original strain. In addition, these variants consist of mutations, which prevents them from being recognized, thereby affecting the current treatment strategies [63].

\section{Future Directions}

The mRNA-based vaccine technology is a promising tool for the development of therapeutic and prophylactic vaccines against novel infectious diseases. Due to the increasing demand for the vaccines, both Pfizer-BioNTech and Moderna Therapeutics have decided to scale up the production of their vaccines. Moderna Therapeutics will be increasing its global production estimate from 500 to 600 million doses this year [67]. Moreover, evaluation of the incidence of asymptomatic COVID-19 cases after administration of the second dose of the vaccines is underway [27]. The participants in the Phase III trial of the Moderna vaccine will be followed up for 2 years after the seconddose administration to assess asymptomatic infection and viral shedding [24]. On 25 February 2021, Pfizer-BioNTech announced that they have initiated clinical trials for evaluating the safety and immunogenicity of a third dose of their BNT162b2 vaccine, to understand its effectiveness against newly emerging variants. A $30 \mu \mathrm{g}$ booster of the vaccine will be administered to participants from the Phase I study in the USA 6-12 months after they have received their initial two doses. In addition, the company is in discussion with the FDA and EMA regarding a clinical study to develop a variant-specific vaccine having a modified mRNA sequence. In this study, a new construct 
of the BNT162b2 vaccine, which is based on the B.1.351 lineage, will be employed. According to Dr. Ugur Sahin, Pfizer-BioNTech is assessing the potential rapid adaptation of the vaccine against the new variants, which might evade the current mRNA vaccine. Moreover, due to the flexibility of their advanced mRNA vaccine platform, booster vaccines can be developed within weeks, which will ensure long-term immunity against the virus, including its new strains [68].

\section{Conclusion}

The interim analysis of the mRNA COVID-19 vaccine trials from Pfizer-BioNtech and Moderna Therapeutics has provided much hope for finding a permanent solution to the current pandemic situation. The efficacy of the BNT162b2 and mRNA-1273 vaccines has been reported as being 95\% and $94.1 \%$, respectively. In addition, both vaccines have been found to be effective against the new strains of the virus. Both companies have now announced their plans of speeding up their manufacturing and updating their vaccines to fight against future variants. Hence, the BNT162b2 and mRNA-1273 vaccines appear to hold promise to turn the tables against the current raging pandemic.

\section{Declarations}

Funding No funding was received for this article.

Conflict of interest The authors do not have any conflict/competing interests.

Ethics approval Not applicable.

Consent to participate Not applicable.

Consent for publication Not applicable.

Availability of data and material Not applicable.

Code availability Not applicable.

\section{References}

1. COVID-19 Map—Johns Hopkins Coronavirus Resource Center. Johns Hopkins Coronavirus Resource Center. 2020. https://coron avirus.jhu.edu/map.html. Accessed 14 Dec 2020.

2. Which countries in Europe are experiencing a second wave of COVID-19? [Internet]. euronews. 2020. https://www.euronews. com/2020/11/18/is-europe-having-a-covid-19-second-wave-count ry-by-country-breakdown. Accessed 14 Dec 2020.

3. Coronavirus Second Wave? Why Cases Increase. 2021. https:// www.hopkinsmedicine.org/health/conditions-and-diseases/coron avirus/first-and-second-waves-of-coronavirus. Accessed $11 \mathrm{Feb}$ 2021.

4. Singh T, Parida S, Lingaraju M, Kesavan M, Kumar D, Singh R. Drug repurposing approach to fight COVID-19. Pharmacol Rep. 2020;72(6):1479-508.

5. Coronavirus disease (COVID-19) advice for the public: When and how to use masks. https://www.who.int/emergencies/diseases/ novel-coronavirus-2019/advice-for-public/when-and-how-to-usemasks. Accessed 14 Dec 2020.

6. COVID-19 vaccines. 2020. https://www.who.int/emergencies/ diseases/novel-coronavirus-2019/covid-19-vaccines. Accessed 17 Dec 2020.

7. Feher J. Quantitative human physiology: Elsevier Academic Press; 2021.

8. mRNA Therapeutics. Translating the message with MRT ${ }^{\mathrm{TM}}$ https://translate.bio/rna-therapeutics/. Accessed 11 Feb 2021.

9. Kowalski PS, Rudra A, Miao L, Anderson DG. Delivering the messenger: advances in technologies for therapeutic mRNA delivery. Mol Ther. 2019;27(4):710-28. https://doi.org/10.1016/j. ymthe.2019.02.012.

10. Zhong Z, Mc Cafferty S, Combes F, et al. mRNA therapeutics deliver a hopeful message. Nano Today. 2018;23:16-39.

11. The co-founder of BioNTech designed the coronavirus vaccine it made with Pfizer in just a few hours over a single day. Business Insider. 2021. https://www.businessinsider.in/science/news/ the-co-founder-of-biontech-designed-the-coronavirus-vaccineit-made-with-pfizer-in-just-a-few-hours-over-a-single-day/artic leshow/79696028.cms. Accessed 11 Feb 2021.

12. How a couple's quest to cure cancer led to the West's first covid19 vaccine. mint. 2021. https://www.livemint.com/news/world/ how-a-couple-s-quest-to-cure-cancer-led-to-the-west-s-firstcovid-19-vaccine-11606999268605.html. Accessed 11 Feb 2021.

13. Wallace-Wells D. We Had the Vaccine the Whole Time. Intelligencer. 2021. https://nymag.com/intelligencer/2020/12/moder na-covid-19-vaccine-design.html. Accessed 11 Feb 2021

14. Jackson N, Kester K, Casimiro D, Gurunathan S, DeRosa F. The promise of mRNA vaccines: a biotech and industrial perspective. NPJ Vaccines. 2020;5(1).

15. Wadhwa A, Aljabbari A, Lokras A, Foged C, Thakur A. Opportunities and challenges in the delivery of mRNA-based vaccines. Pharmaceutics. 2020;12(2):102.

16. Pardi N, Hogan M, Porter F, Weissman D. mRNA vaccines-a new era in vaccinology. Nat Rev Drug Discov. 2018;17(4):261-79.

17. Zeng C, Zhang C, Walker PG, Dong Y. formulation and delivery technologies for mRNA vaccines. 2020. In. Current Topics in microbiology and immunology. Springer, Berlin, Heidelberg. https://doi.org/10.1007/82_2020_217.

18. Pfizer-BioNTech COVID-19 vaccines (BNT162, PF-07302048) and related biological products advisory committee briefing document-FDA.gov. Accessed 11 Feb 2021.

19. Why Moderna may have an edge in the vaccine race: refrigeration. https://www.nationalgeographic.com/science/2020/11/moder na-edges-pfizer-coronavirus-efficacy-and-refrigeration. Accessed 17 Dec 2020.

20. Pfizer and BioNTech Conclude Phase 3 Study of COVID-19 Vaccine Candidate, Meeting All Primary Efficacy Endpoints. https://www.pfizer.com/news/press-release/press-release-detail/ pfizer-and-biontech-conclude-phase-3-study-covid-19-vaccine. Accessed 11 Feb 2021.

21. Hodgson SH, Mansatta K, Mallett G, Harris V, Emary KR, Pollard AJ. What defines an efficacious COVID-19 vaccine? A review of the challenges assessing the clinical efficacy of vaccines against SARS-CoV-2. Lancet Infect Dis. 2020;27.

22. Baden LR, El Sahly HM, Essink B, Kotloff K, Frey S, Novak R, Diemert D, Spector SA, Rouphael N, Creech CB, McGettigan J. 
Efficacy and safety of the mRNA-1273 SARS-CoV-2 vaccine. N Engl J Med. 2021;384:403-16. https://doi.org/10.1056/NEJMo a2035389.

23. Corbett KS, Flynn B, Foulds KE, Francica JR, Boyoglu-Barnum S, Werner AP, Flach B, O'Connell S, Bock KW, Minai M, Nagata BM. Evaluation of the mRNA-1273 vaccine against SARS-CoV-2 in nonhuman primates. N Engl J Med. 2020;383(16):1544-55.

24. Jackson LA, Anderson EJ, Rouphael NG, Roberts PC, Makhene M, Coler RN, McCullough MP, Chappell JD, Denison MR, Stevens LJ, Pruijssers AJ. An mRNA vaccine against SARS-CoV-2preliminary report. N Engl J Med. 2020;383(20):1920-31.

25. Mulligan MJ, Lyke KE, Kitchin N, Absalon J, Gurtman A, Lockhart S, Neuzil K, Raabe V, Bailey R, Swanson KA, Li P. Phase I/ II study of COVID-19 RNA vaccine BNT162b1 in adults. Nature. 2020;586(7830):589-93.

26. Walsh EE, Frenck Jr RW, Falsey AR, Kitchin N, Absalon J, Gurtman A, Lockhart S, Neuzil K, Mulligan MJ, Bailey R, Swanson KA. Safety and immunogenicity of two RNA-based Covid-19 vaccine candidates. N Engl J Med. 2020.

27. Polack FP, Thomas SJ, Kitchin N, Absalon J, Gurtman A, Lockhart S, Perez JL, Pérez Marc G, Moreira ED, Zerbini C, Bailey R. Safety and efficacy of the BNT162b2 mRNA covid-19 vaccine. N Engl J Med. 2020.

28. Nikolai LA, Meyer CG, Kremsner PG, Velavan TP. Asymptomatic SARS Coronavirus 2 infection: invisible yet invincible. Int J Infect Dis. 2020.

29. Mirasol F. Meeting the Challenges for Scaling Up Vaccine Manufacturing Systems to Supply Adequate Capacity. BioPharm International. 2021. https://www.biopharminternational.com/view/ meeting-the-challenges-for-scaling-up-vaccine-manufacturingsystems. Accessed 12 Feb 2021.

30. Pfizer and BioNTech, scaling up for $2 \mathrm{~B}$ coronavirus vaccine doses, temporarily cut deliveries in EU, Canada. FiercePharma. 2021. https://www.fiercepharma.com/pharma/pfizer-and-biont ech-pushing-for-2b-coronavirus-vaccine-doses-2021-temporarily-reduce. Accessed 12 Feb 2021.

31. Lonza and Moderna shoot for billion COVID-19 vaccine doses. https://www.biopharma-reporter.com/Article/2020/ 05/05/Lonza-and-Moderna-partner-for-COVID-19-vaccine. Accessed 14 Feb 2021

32. Chitravanshi S. Pfizer working on cold storage issues to ensure temperature integrity. https://www.business-standard.com/artic le/companies/pfizer-working-on-cold-storage-issues-to-ensuretemperature-integrity-120120700024_1.html. Accessed 14 Feb 2021.

33. Reuters S. Moderna's Covid vaccine $94.5 \%$ effective, edges over Pfizer on storage. https://www.business-standard.com/article/ companies/moderna-s-covid-vaccine-94-5-effective-edgesover-pfizer-on-storage-120111601113_1.html. Accessed 14 Feb 2021.

34. Pfizer and BioNTech Reach Agreement with COVAX for Advance Purchase of Vaccine to Help Combat COVID-19. https://www.pfizer.com/news/press-release/press-release-detail/ pfizer-and-biontech-reach-agreement-covax-advance-purchase. Accessed 14 Feb 2021

35. Moderna agreed to 'equitable access' for its coronavirus vaccine, but most of its doses are going to wealthy countries. https://www.washingtonpost.com/world/coronavirus-vaccineaccess-poor-countries-moderna/2021/02/12/0586e532-671211eb-bf81-c618c88ed605_story.html. Accessed 14 Feb 2021.

36. COVID-19 Vaccine: Will It Protect Against New Variants And Do You Need A 2nd Dose? https://www.npr.org/sections/healthshots/2021/02/02/963047878/covid-19-vaccine-will-it-protectagainst-new-variants-and-do-you-need-a-2nd-dose. Accessed 12 Feb 2021.
37. The Moderna COVID-19 (mRNA-1273) vaccine: what you need to know. https://www.who.int/news-room/feature-stories/detail/ the-moderna-covid-19-mrna-1273-vaccine-what-you-need-toknow. Accessed 12 Feb 2021.

38. Pfizer, Moderna vaccines have reduced effectiveness against South African variant, studies show. https://www.washington post.com/nation/2021/02/18/coronavirus-covid-live-updatesus/. Accessed 12 Feb 2021.

39. Moderna COVID-19 Vaccine Retains Neutralizing Activity Against Emerging Variants First Identified in the U.K. and the Republic of South Africa. https://investors.modernatx.com/ node/10841/pdf. Accessed 12 Feb 2021.

40. New Variants of Coronavirus: What You Should Know. https:// www.hopkinsmedicine.org/health/conditions-and-diseases/ coronavirus/a-new-strain-of-coronavirus-what-you-shouldknow. Accessed 14 Feb 2021.

41. Hobernik D, Bros M. DNA vaccines-how far from clinical use? Int J Mol Sci. 2018;19(11):3605.

42. mRNA vaccines: Disruptive innovation in vaccination. https:// www.modernatx.com/sites/default/files/RNA_Vaccines_White_ Paper_Moderna_050317_v8_4.pdf. Accessed 14 Feb 2021.

43. Understanding and Explaining mRNA COVID-19 Vaccines. https://www.cdc.gov/vaccines/covid-19/hcp/mrna-vaccinebasics.html. Accessed 17 Dec 2020

44. Verbeke R, Lentacker I, De Smedt SC, Dewitte H. Three decades of messenger RNA vaccine development. Nano Today. 2019;28:

45. Coronavirus Vaccine Tracker. https://www.nytimes.com/inter active/2020/science/coronavirus-vaccine-tracker.html. Accessed 14 Feb 2021.

46. Bloom K, van den Berg F, Arbuthnot P. Self-amplifying RNA vaccines for infectious diseases. Gene Ther. 2020;22:1-3.

47. mRNA Vaccines. Gennova Biopharmaceuticals Limited. 2020. https://gennova.bio/mrna-vaccines/. Accessed 16 Dec 2020.

48. Pfizer and BioNTech Granted FDA Fast Track Designation for Two Investigational mRNA-based Vaccine Candidates against SARS-CoV-2. https://www.pfizer.com/news/press-release/pressrelease-detail/pfizer-and-biontech-granted-fda-fast-track-desig nation-two. Accessed 8 Dec 2020.

49. Pfizer and BioNTech Achieve First Authorization in the World for a Vaccine to Combat COVID-19. https://www.pfizer.com/news/ press-release/press-release-detail/pfizer-and-biontech-achievefirst-authorization-world. Accessed 14 Feb 2021.

50. Business Today.In. 2020. After UK, Bahrain Approves PfizerBioNtech Vaccine For Emergency Use. [online]. https://www. google.com/amp/s/m.businesstoday.in/lite/story/after-uk-bahra in-approves-pfizer-biontech-vaccine-for-emergency-use/1/423983. html. Accessed 10 Dec 2020.

51. USA TODAY. 2020. Coronavirus Updates: 'Incalculable Loss' Of Life In Los Angeles County; Pennsylvania Gov. Tom Wolf Tests Positive; Canada Oks Pfizer Vaccine. [online]. https://amp.usato day.com/amp/6499047002. Accessed 12 Dec 2020.

52. M.businesstoday.in. 2020. Pfizer Vaccine: UK Regulator Allays Fears After 4 Bell's Palsy Cases In US Trials. [online]. https://m. businesstoday.in/story/pfizer-vaccine-uk-regulator-allays-fearsafter-4-bells-palsy-cases-in-us-trials/1/424382.html. Accessed 12 Dec 2020.

53. Size F, Size F, WIRE B. Pfizer and BioNTech Celebrate Historic First Authorization in the U.S. of Vaccine to Prevent COVID19. https://www.businesswire.com/news/home/20201211005640/ en/. Accessed 12 Dec 2020.

54. Emergency Use Authorization (EUA) for an Unapproved Product Review Memorandum. https://www.fda.gov/media/144416/downl oad. Accessed 14 Feb 2021. 
55. Roche partners with Moderna to include SARS-CoV-2 antibody test in ongoing COVID-19 vaccine trials. https://www.roche.com/ media/releases/med-cor-2020-12-09.htm.

56. Moderna Announces Longer Shelf Life for its COVID-19 Vaccine Candidate at Refrigerated Temperatures. https://investors.moder natx.com/news-releases/news-release-details/moderna-annou nces-primary-efficacy-analysis-phase-3-cove-study. Accessed 16 Feb 2021.

57. Japan approves Pfizer's COVID-19 vaccine. https://japantoday. com/category/national/Japan-approves-Pfizer's-COVID-19-vacci ne. Accessed 16 Feb 2021.

58. Covid-19 vaccine update: Japan expected to approve Pfizer vaccine. https://www.financialexpress.com/lifestyle/health/covid-19vaccine-update-japan-expected-to-approve-pfizer-vaccine/21937 40/. Accessed 16 Feb 2021.

59. 0 virus deaths reported from 520,000 given the Pfizer vaccine in Israel. Hardly anybody even got sick, suggesting $93 \%$ effectiveness. https://www.businessinsider.in/science/health/news/pfize rs-vaccine-is-93-effective-israeli-healthcare-giant-says-it-repor ted-0-deaths-and-4-severe-cases-from-520000-vaccinated-peopl e-/articleshow/80884583.cms. Accessed 16 Feb 2021.

60. Qatar approves emergency use of Moderna's COVID-19 vaccine. https://www.aljazeera.com/news/2021/2/10/qatar-approves-emerg ency-use-for-modernas-covid-19-vaccine. Accessed 16 Feb 2021.

61. Moderna Gets Added to Southeast Asia's Vaccine Arsenal. https:// www.bloomberg.com/news/articles/2021-02-08/moderna-getsadded-to-arsenal-southeast-asia-vaccine-tracker. Accessed 16 Feb 2021.

62. In huge boost, FDA says it will now allow Moderna to increase vaccine in each vial. https://thehill.com/changing-america/ well-being/prevention-cures/538640-in-huge-boost-fda-says-itwill-now-allow-moderna. Accessed 16 Feb 2021.

63. COVID-19 and your health. Centers for Disease Control and Prevention 2021. https://www.cdc.gov/coronavirus/2019-ncov/trans mission/variant.html. Accessed 14 Feb 2021.

64. How symptoms of new coronavirus strains different from original one [Internet]. Business-standard.com 2021. https://www.busin ess-standard.com/article/current-affairs/how-symptoms-of-newcoronavirus-strains-different-from-original-one-121021701316_1. html. Accessed 16 Feb 2021.

65. Serum institute may alter covid shot to tackle mutant strain. https://www.livemint.com/news/india/sii-may-have-to-adjustcovid-vaccine-composition-for-south-african-variant-1161288322 6791.html. Accessed 15 Feb 2021.

66. COVID-19 South African, Brazilian variants detected in India: Govt. https://www.indiatvnews.com/news/india/covid19-southafrican-brazilian-variants-detected-in-india-coronavirus-new-strai ns-icmr-latest-news-685338. Accessed 15 Feb 2021.

67. Moderna Provides COVID-19 Vaccine Supply Update. https:// investors.modernatx.com/news-releases/news-release-details/ moderna-provides-covid-19-vaccine-supply-update. Accessed 16 Feb 2021.

68. Pfizer and BioNTech Initiate a Study as Part of Broad Development Plan to Evaluate COVID-19 Booster and New Vaccine Variants. https://www.pfizer.com/news/press-release/press-relea se-detail/pfizer-and-biontech-initiate-study-part-broad-devel opment. Accessed 27 Feb 2021. 